\title{
Comparative study of the electron- and positron-atom bremsstrahlung
}

\author{
V. A. Yerokhin, ${ }^{1,2,3}$ A. Surzhykov, ${ }^{1,2}$ R. Märtin, ${ }^{4,2}$ S. Tashenov, ${ }^{1}$ and G. Weber ${ }^{4,2}$ \\ ${ }^{1}$ Institute of Physics, Heidelberg University, Im Neuenheimer Feld 226, D-69120 Heidelberg, Germany \\ ${ }^{2}$ GSI Helmholtzzentrum für Schwerionenforschung, Planckstraße 1, D-64291 Darmstadt, Germany \\ ${ }^{3}$ Center for Advanced Studies, St. Petersburg State Polytechnical University, \\ Polytekhnicheskaya 29, St. Petersburg 195251, Russia \\ ${ }^{4}$ Helmholtz-Institut Jena, Fröbelstieg 3, D-07743 Jena, Germany
}

\begin{abstract}
Fully relativistic treatment of the electron-atom and positron-atom bremsstrahlung is reported. The calculation is based on the partial-wave expansion of the Dirac scattering states in an external atomic field. A comparison of the electron and positron bremsstrahlung is presented for the single and double differential cross sections and the Stokes parameters of the emitted photon. It is demonstrated that the electron-positron symmetry of the bremsstrahlung spectra, which is nearly exact in the nonrelativistic regime, is to a large extent removed by the relativistic effects.
\end{abstract}

PACS numbers: 34.80.-i, 34.50.-s, 41.60.-m, 78.70.En

When a charged particle traverses an atomic field, a part of its energy may be converted into radiation. This is the atomic bremsstrahlung, one of the fundamental collision processes. It is one of the important mechanisms of the energy loss in hot plasmas and in particle beams traversing thick targets. The process of the electron-atom bremsstrahlung has been extensively studied in the literature. The single and double differential cross sections of this process were tabulated [1, 2] and the polarization properties of the emitted radiation were calculated $[3[6]$.

Much less is known on the positron-atom bremsstrahlung. Several reported investigations of this process [7 9] were dealing primarily with the bremsstrahlung energy loss. It was shown that, for high energies of the incident and final projectile, the cross sections of the electron and positron bremsstrahlung are very much similar. However, when the energy of the final projectile decreases, the cross section of the positron bremsstrahlung becomes increasingly suppressed as compared to the electron one, because of the strong Coulomb repulsion between the positron and nucleus at short distances. The ratio of the positron-to-electron bremsstrahlung stopping power was the main subject of those early works.

To the best of our knowledge, the angular dependence of the cross section and the polarization properties of the relativistic positron-atom bremsstrahlung have never been studied. They are becoming subjects of experimental interest today, with the advent of techniques for the production of highly polarized positrons beams [10] and the detection of polarization correlations in the bremsstrahlung radiation [11, 12].

In the present investigation, we make a comparative study of the positron- and electron-atom bremsstrahlung, by analyzing the double differential cross section and the polarization correlations between the incident projectile and the emitted photon. The calculation is performed within the fully relativistic approach based on the partialwave representation of the Dirac continuum states with a fixed value of the asymptotic momentum. This work extends our previous calculations of the electron-atom bremsstrahlung [5, 13].

\section{THEORY}

Relativistic theory of the electron-atom bremsstrahlung is nowadays well established and can be found, e.g., in the review article 14]. For our bremsstrahlung calculations we use the density-matrix formalism described in detail in our previous paper [5]. In this section, we will extend the formulas obtained previously to the case of the positron bremsstrahlung. Such extension can be done by exploiting the fact that in the QED theory, an incoming positron with a four-momentum $p_{i}$ and a helicity $m_{i}$ can be described as an outgoing electron with a four-momentum $-p_{i}$ and a helicity $-m_{i}$ (see, e.g., books [15, 16]).

We now consider the transition from the electron-atom to the positron-atom bremsstrahlung in more detail. The amplitude of the electron-atom bremsstrahlung is

$$
\begin{aligned}
M_{i f}^{\mathrm{el}}(\lambda)= & \int d \boldsymbol{r} \Psi^{(+)^{\dagger}}\left(\varepsilon_{i}, \boldsymbol{p}_{i}, m_{i}\right) \\
& \times \boldsymbol{\alpha} \cdot \hat{\boldsymbol{u}}_{\lambda} e^{i \boldsymbol{k} \cdot \boldsymbol{r}} \Psi^{(-)}\left(\varepsilon_{f}, \boldsymbol{p}_{f}, m_{f}\right),
\end{aligned}
$$

where $\Psi^{(+)}\left(\varepsilon_{i}, \boldsymbol{p}_{i}, m_{i}\right)$ is the wave function of the inital electron state with the energy $\varepsilon_{i}$, the momentum $\boldsymbol{p}_{i}$, the helicity $m_{i}$, and the asymptotics of an outgoing spherical wave, and $\Psi^{(-)}\left(\varepsilon_{f}, \boldsymbol{p}_{f}, m_{f}\right)$ is the wave function of the final electron state with the energy $\varepsilon_{f}$, the momentum $\boldsymbol{p}_{f}$, the helicity $m_{f}$, and the asymptotics of an incoming spherical wave, and $\boldsymbol{k}$ and $\lambda$ are the momentum and polarization of the emitted photon, respectively. The Dirac scattering states $\Psi^{( \pm)}(\varepsilon, \boldsymbol{p}, m)$ are given by their partialwave expansion [17, 18]

$$
\Psi^{( \pm)}(\varepsilon, \boldsymbol{p}, m)=\frac{1}{\sqrt{p|\varepsilon|}} \sum_{\kappa \mu} i^{l} e^{ \pm i \Delta_{\kappa}} C_{l m_{l}, \frac{1}{2} m}^{j \mu} Y_{l m_{l}}^{*}(\hat{\boldsymbol{p}})|\varepsilon \kappa \mu\rangle
$$

where $|\varepsilon \kappa \mu\rangle$ are the Dirac continuum states with a given 
angular-momentum quantum number $\kappa$ and angularmomentum projection $\mu, j=|\kappa|-1 / 2, l=|\kappa+1 / 2|-1 / 2$, and $\Delta_{\kappa}=\sigma_{\kappa}-\sigma_{\kappa}^{(0)}$ is the difference between the asymptotic large-distance phase of the Dirac-Coulomb solution and the free Dirac solution (see book [17] for details).

In order to obtain the amplitude of the positron-atom bremsstrahlung from Eq. (1), we need to make the following substitutions in the wave functions: $\varepsilon \rightarrow-\varepsilon$, $\boldsymbol{p} \rightarrow-\boldsymbol{p}, m \rightarrow-m, \Psi^{( \pm)} \rightarrow \Psi^{(\mp)}$, and to interchange the initial and the final state. The resulting amplitude of the positron-atom bremsstrahlung is given by

$$
\begin{aligned}
M_{i f}^{\mathrm{pos}}(\lambda)= & \int d \boldsymbol{r} \Psi^{(+)^{\dagger}}\left(-\varepsilon_{f},-\boldsymbol{p}_{f},-m_{f}\right) \\
& \times \boldsymbol{\alpha} \cdot \hat{\boldsymbol{u}}_{\lambda} e^{i \boldsymbol{k} \cdot \boldsymbol{r}} \Psi^{(-)}\left(-\varepsilon_{i},-\boldsymbol{p}_{i},-m_{i}\right),
\end{aligned}
$$

where $\boldsymbol{p}_{i}\left(\boldsymbol{p}_{f}\right)$ and $m_{i}\left(m_{f}\right)$ are the momentum and helicity of the initial (final) state positron, respectively.

Assuming that the final-state positron is not observed in the experiment, we introduce the two-by-two reduced density matrix of the final (photon) state,

$$
\begin{aligned}
\left\langle\boldsymbol{k} \lambda\left|\rho_{f}\right| \boldsymbol{k} \lambda^{\prime}\right\rangle= & \sum_{m_{i} m_{i^{\prime}} m_{f}} \int d \Omega_{f} M_{i f}^{*}(\lambda) M_{i^{\prime} f}\left(\lambda^{\prime}\right) \\
& \times\left\langle\boldsymbol{p}_{i} m_{i}\left|\rho_{i}\right| \boldsymbol{p}_{i} m_{i^{\prime}}\right\rangle
\end{aligned}
$$

where $\Omega_{f}$ is the solid angle of the scattered positron, and $\left\langle\boldsymbol{p}_{i} m_{i}\left|\rho_{i}\right| \boldsymbol{p}_{i} m_{i^{\prime}}\right\rangle$ is the density matrix of the initial positron state. Note that the inital density matrix is the same for positrons and electrons (since it depends only on the spin of the particle and not on its charge). The photon direction $\hat{\boldsymbol{k}}=\boldsymbol{k} / \boldsymbol{k}$ will be characterized by the Euler angles $(\theta, \phi)$, with the $z$ axis directed along the momentum of the initial-state projectile $\boldsymbol{p}_{i}$. The final-state density matrix (44) contains all the information needed for calculating the differential cross section and the polarization correlations of the bremsstrahlung radiation.

Extending the derivation given in Ref. [5] to the case of the positron-atom bremsstrahlung, we obtain

$$
\begin{aligned}
\left\langle\boldsymbol{k} \lambda\left|\rho_{f}\right| \boldsymbol{k} \lambda^{\prime}\right\rangle= & 8(2 \pi)^{4} \sum_{\kappa_{i} \kappa_{i}^{\prime} \kappa_{f}} \sum_{L L^{\prime} \kappa g t} \sum_{\gamma_{1} \gamma_{2}} D_{\gamma_{1} \gamma_{2}}^{g^{*}}(\hat{\boldsymbol{k}})(-1)^{\kappa} \rho_{\kappa, \gamma_{1}}^{(i)} i^{l_{i}-l_{i}^{\prime}-L+L^{\prime}} e^{i \Delta_{\kappa_{i},-\varepsilon_{i}}-i \Delta_{\kappa_{i}^{\prime},-\varepsilon_{i}}}\left[L, L^{\prime}, j_{i}, j_{i}^{\prime}, l_{i}, l_{i}^{\prime}, g, \kappa\right]^{1 / 2} \\
& \times(-1)^{j_{i}^{\prime}-j_{f}+l_{i}+g+\kappa} C_{L^{\prime}-\lambda^{\prime}, L \lambda}^{g \gamma_{2}} C_{l_{i} 0, l_{i}^{\prime} 0}^{t 0} C_{g-\gamma_{1}, \kappa \gamma_{1}}^{t 0}\left\{\begin{array}{ccc}
L & j_{f} & j_{i} \\
j_{i}^{\prime} & g & L^{\prime}
\end{array}\right\}\left\{\begin{array}{ccc}
1 / 2 & 1 / 2 & \kappa \\
j_{i}^{\prime} & j_{i} & g \\
l_{i}^{\prime} & l_{i} & t
\end{array}\right\} \\
& \times \sum_{p p^{\prime}}(i \lambda)^{p}\left(-i \lambda^{\prime}\right)^{p^{\prime}}\left\langle-\varepsilon_{i} \kappa_{i}\left\|\boldsymbol{\alpha} \cdot \boldsymbol{a}_{L}^{(p)}\right\|-\varepsilon_{f} \kappa_{f}\right\rangle\left\langle-\varepsilon_{i} \kappa_{i}^{\prime}\left\|\boldsymbol{\alpha} \cdot \boldsymbol{a}_{L^{\prime}}^{\left(p^{\prime}\right)}\right\|-\varepsilon_{f} \kappa_{f}\right\rangle^{*}
\end{aligned}
$$

where $D_{M \lambda}^{L}$ is Wigner's $D$ function [19], $\rho_{\kappa}^{(i)}$ is the spherical tensor of the initial-state density matrix [see Eq. (10) of Ref. [5]], $\boldsymbol{a}_{L}^{(p)}$ are the magnetic $(p=0)$ and electric $(p=1)$ operators defined by Eqs. (15)-(17) of Ref. [5], $\left[x_{1}, x_{2}, \ldots\right] \equiv\left(2 x_{1}+1\right)\left(2 x_{2}+1\right) \ldots$ The states $\left|-\varepsilon_{i} \kappa_{i}\right\rangle$ and $\left|-\varepsilon_{f} \kappa_{f}\right\rangle$ are the spherical-wave continuum-state Dirac wave functions with a negative energy, $-\varepsilon_{i}<-m$ and $-\varepsilon_{f}<-m$, where $m$ is the electron rest mass.

In our calculations of the final-state density matrix, we used the method described in Ref. [5], with radial integrals evaluted numerically and the integration contour rotated to the imaginary axis. We performed calculations for two types of the target, (i) the bare nucleus and (ii) the neutral atom. In the latter case, the electronic structure of the atom was represented by a static screening potential obtained by the Dirac-Fock method.

The negative-energy continuum-state Dirac wave functions $|-\varepsilon \kappa\rangle$ for the point Coulomb potential can be calculated by using their analytic representation in terms of the Whittaker $M$ function [18], similarly to that for the positive energies. For the neutral atoms, however, the wave functions have to be computed numerically. In this case, it is convenient to transform the negative-energy states to the positive-energy ones by using the symmetry of the radial Dirac equation. One can show that the upper and lower radial components of the negative-energy Dirac solution with the potential $V, g_{-\varepsilon, \kappa}^{V}$ and $f_{-\varepsilon, \kappa}^{V}$, can be expressed in terms of the components of the positiveenergy solutions with the potential $-V$ as

$$
\begin{aligned}
& g_{-\varepsilon, \kappa}^{V}(r)=f_{\varepsilon,-\kappa}^{-V}(r), \\
& f_{-\varepsilon, \kappa}^{V}(r)=g_{\varepsilon,-\kappa}^{-V}(r) .
\end{aligned}
$$

This approach to the evaluation of the negative-energy Dirac states was previously used in Ref. [20].

\section{RESULTS AND DISCUSSION}

We begin with calculating the single-differential cross section of the electron and positron-atom bremsstrahlung. The results can be conveniently represented in terms of the scaled cross section $\sigma \equiv$ $\left(k / Z^{2}\right) d \sigma / d k$, where $Z$ is the nuclear charge and $k$ is the photon energy. This part of our calculations can 
be compared with the previous work by Feng, Pratt, and Tseng [8]. Very good agreement with their calculation was found. So, for the inital kinetic energy $E=50 \mathrm{keV}$, the fractional energy carried by the photon $k / E=0.6$, and the bare-nucleus target, we obtain the ratio of the positron-to-electron bremsstrahlung cross sections $\sigma^{+} / \sigma^{-}=0.61498$ for $Z=8$ and 0.003839 for $Z=92$, whereas Ref. [8] reports 0.615 and 0.00384 , respectively.

Our numerical results for the cross sections of the positron and electron bremsstrahlung are presented in Fig. 1 for two targets, carbon and gold, and the initial projectile energy of $100 \mathrm{keV}$. Carbon is an essentially nonrelativistic system. In this case, the positron bremsstrahlung is only slightly suppressed as compared to the electron one and the difference between the bare nucleus and neutral atom is very small (i.e., the screening effect of the atomic electrons is nearly negligible). For gold, on the contrary, the relativistic binding effects are large. Within the classical-physics picture, one can expect that the strong Coulomb potential of the nucleus significantly changes the projectile velocity at the point of the closest approach (where the photon emission is most probable) and thus breaks the symmetry between the electron and the positron spectra. Indeed, our calculations for gold show a large suppression of the positron bremsstrahlung in the region $k / E>0.5$ and also a significant screening effect of the target electrons. We observe that the screening effect reduces the cross section for the case of the electron bremsstrahlung but enhances it for the positron bremsstrahlung. Remarkably, in the region $k / E \sim 1$, the screening effect enhances the positron bremsstrahlung by an order of magnitude as compared to the pure Coulomb field (as noted already in Ref. [8]). But, as the resulting cross section is still very small, the effect is difficult to observe experimentally.

The numerical results for the double-differential cross section, $d \sigma \equiv\left(k / Z^{2}\right) d \sigma /\left(d k d \Omega_{k}\right)$, are presented in Fig. 2. The calculation was performed for neutral atomic targets (carbon and gold) and several energies of the incoming projectile. We observe that the dominant difference between the electron $d \sigma^{-}$and positron $d \sigma^{+}$ cross sections comes from the suppression of the positron bremsstrahlung. As can be seen from the picture, the suppression grows with increasing the nuclear charge and decreasing the energy of the initial projectile. This is in agreement with Ref. [9], which concludes that, for a large range of the kinetic energy of the incoming projectile $E_{\text {kin }}$, the overall suppression factor is a nearly linear function of $Z^{2} / E_{\text {kin }}$.

Next, we compare the polarization properties of the electron and positron bremsstrahlung. The most important polarization property is the Stokes parameter of the emitted photon $P_{1}$ for the initially unpolarized projectile. (In this case, $P_{1}$ yields also the degree of the linear polarization of the emitted radiation.) The numerical results for $P_{1}$ are presented in Figs. 3 and 4 . Since the screening effect of atomic electrons on the Stokes param- eters is rather weak, the results shown were obtained for the bare nucleus targets. We again observe that for the light targets (carbon), the polarization of the positron bremsstrahlung is almost identical to the electron one. For the gold target, however, the relativistic effects break the electron-positron symmetry of the bremsstrahlung radiation. The difference between the electron and positron bremsstrahlung spectra becomes increasingly more pronounced when the initial projectile energy is enlarged.

The second polarization correlation that is of the experimental interest today is the Stokes parameter of the emitted photon $P_{2}$. It manifests itself as a rotation of the polarization ellipse of the emitted radiation in the plane perpendicular to the photon momentum. The ratio of $P_{2}$ and $P_{1}$ yields the tilt angle $\chi$ of the polarization ellipse $\left(P_{2} / P_{1}=\tan 2 \chi\right)$, which can nowadays be measured very precisely [11]. Our numerical results for the Stokes parameter $P_{2}$ for the initially longitudinally polarized projectile are presented in Fig. 5 Since $P_{2}$ is purely a relativistic effect, its typical numerical values are very small for low- $Z$ target but grow rapidly when the initial projectile energy and the nuclear charge are increased.

An important observation is that the numerical values of $P_{2}$ for the initially longitudinally polarized electrons and positrons are of the opposite sign. This could have been anticipated from the fact that $P_{2}$ scales linearly with $Z$ [4] and, therefore, changes its sign after the substitution $Z \rightarrow-Z$. The consequence of the opposite sign of $P_{2}\left(e^{-}\right)$and $P_{2}\left(e^{+}\right)$is that the rotation of the polarization ellipse of the emitted radiation for the longitudinally polarized electron and positron beams will occur in the opposite directions.

Beside this effect, we observe that $P_{2}\left(e^{-}\right)$and $-P_{2}\left(e^{+}\right)$ nearly coincide for light targets but become increasingly different as the nuclear charge and the initial projectile energy are enlarged. We also note that the difference between the Stokes parameters is generally smaller at the forward angles and larger at the backward angles. This effect can be understood from simple classical-physics arguments. In order to scatter backwards off the positivelycharge nucleus, the electron has to go all the way around the nucleus, whereas the positron just enters the nuclear field and bounces back. So, for the back scattering, the paths within the region of the strong field (and, therefore, the accumulated relativistic effects) are very different for the electron and the positron. For the forward scattering, however, mainly the straight trajectories contribute and so the paths of the electron and the positron in the strong Coulomb field are almost the same.

In summary, we perfomed a calculation of the electronatom and positron-atom bremsstrahlung within the rigorous relativistic approach based on the partial-wave expansion of the Dirac wave functions in the external atomic field. Comparison between the electron and positron bremsstrahlung spectra is made for the single and double differential cross sections and the Stokes parameters of the emitted radiation. It is demonstrated that for the low- $Z$ targets, the polarization of the elec- 
tron and positron bremsstrahlung radiation is very much similar (except for the polarization correlations vanishing in the nonrelativistic limit). However, for heavy relativistic targets and high impact energies, the positron bremsstrahlung becomes significantly suppressed and distorted as compared to the electron bremsstrahlung.
The work reported in this paper was supported by the Helmholtz Gemeinschaft (Nachwuchsgruppe VHNG-421). S.T. acknowledges the support by the German Research Foundation (DFG) within the Emmy Noether program under contract No. TA 740 1-1.
[1] R. H. Pratt, H. K. Tseng, C. M. Lee, and L. Kissel, At. Data Nucl. Data Tables 20, 175 (1977).

[2] L. Kissel, C. A. Quarles, and R. H. Pratt, At. Data Nucl. Data Tables 28, 381 (1983).

[3] H. K. Tseng and R. H. Pratt, Phys. Rev. A 3, 100 (1971).

[4] H. K. Tseng and R. H. Pratt, Phys. Rev. A 7, 1502 (1973).

[5] V. A. Yerokhin and A. Surzhykov, Phys. Rev. A 82, 062702 (2010).

[6] D. H. Jakubassa-Amundsen, Phys. Rev. A 82, 042714 (2010).

[7] R. J. Jabbur and R. H. Pratt, Phys. Rev. 129, 184 (1963).

[8] I. J. Feng, R. H. Pratt, and H. K. Tseng, Phys. Rev. A 24, 1358 (1981).

[9] L. Kim, R. H. Pratt, S. M. Seltzer, and M. J. Berger, Phys. Rev. A 33, 3002 (1986).

[10] G. Alexander et al., Phys. Rev. Lett. 100, 210801 (2008).

[11] S. Tashenov, T. Bäck, R. Barday, B. Cederwall, J. Enders, A. Khaplanov, Y. Poltoratska, K.-U. Schässburger, and A. Surzhykov, Phys. Rev. Lett. 107, 173201 (2011).

[12] R. Märtin et al., Phys. Rev. Lett. 108, 264801 (2012).

[13] G. Weber, R. Märtin, A. Surzhykov, M. Yasuda, V. A.
Yerokhin, and Th. Stöhlker, Nucl. Instr. Meth. Res. B 279, 155 (2012).

[14] R. H. Pratt and I. J. Feng, The electron bremsstrahlung spectrum from neutral atoms and ions, In: C. F. Barnett and M. F. Harrison (eds.), in Applied Collision Physics. Academic Press, NY, 1984.

[15] J. D. Bjorken and S. D. Drell, Relativistic Quantum Fields, McGraw-Hill, NY, 1965.

[16] C. Itzykson and J. Bernard Zuber, Quantum Field Theory, McGraw-Hill, NY, 1980.

[17] M. E. Rose, Relativistic Electron Theory, John Wiley, NY, 1961.

[18] J. Eichler and W. Meyerhof, Relativistic Atomic Collisions, Academic Press, San Diego, 1995. Note the misprinted overall sign in Eq. (4.115).

[19] D. A. Varshalovich, A. N. Moskalev, and V. K. Khersonskï, Quantum Theory of Angular Momentum, World Scientific, Singapure, 1988.

[20] A. N. Artemyev, T. Beier, J. Eichler, A. E. Klasnikov, C. Kozhuharov, V. M. Shabaev, T. Stöhlker, and V. A. Yerokhin, Phys. Rev. A 67, 052711 (2003). 

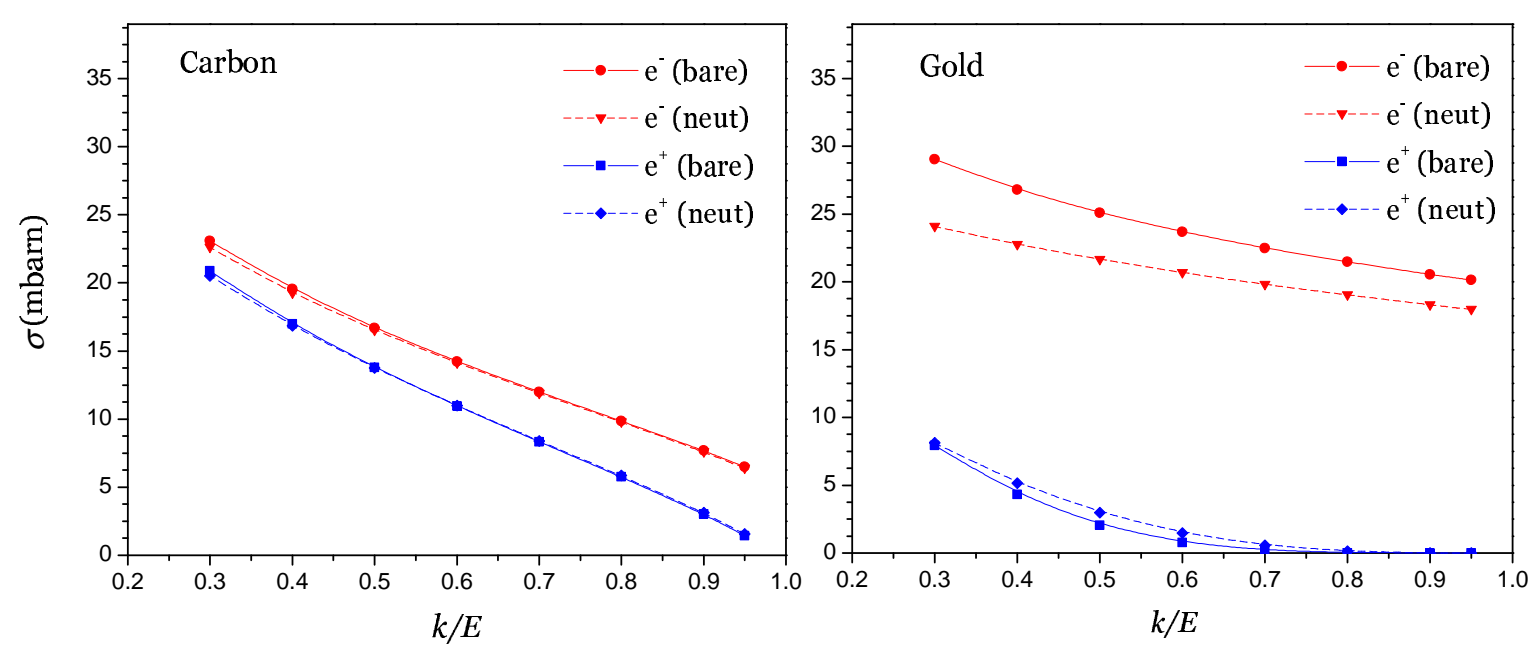

FIG. 1: (Color online) Single-differential cross section $\sigma \equiv\left(k / Z^{2}\right) d \sigma / d k$ for the initially unpolarized electrons $\left(e^{-}\right.$, red) and positrons $\left(e^{+}\right.$, blue), for the carbon (left) and gold (right) targets, as a function of the fractional energy carried by the photon. The initial energy of projectile is fixed by $E=100 \mathrm{keV}$. Calculational results for the bare nucleus are shown by solid line and those for the neutral atom, by the dashed line.
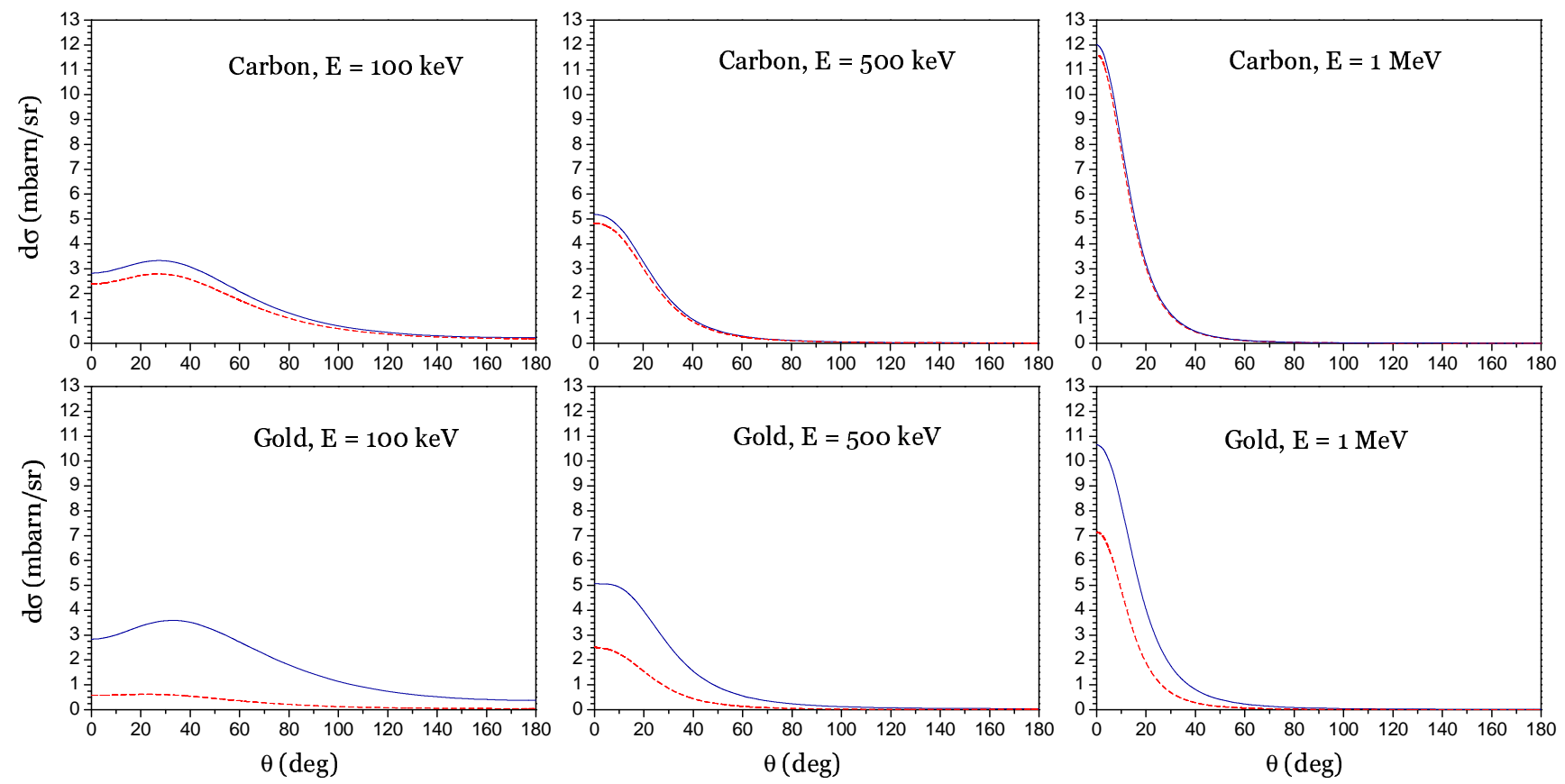

FIG. 2: (Color online) Double-differential cross section $d \sigma \equiv\left(k / Z^{2}\right) d \sigma /\left(d k d \Omega_{k}\right)$ for the initially unpolarized electrons (blue, solid line) and positrons (red, dashed line), for the carbon (top) and gold (bottom) targets, as a function of the photon emission angle, for different initial energies of the projectile. The fractional energy carried by photon is $k / E=0.5$. The calculation was performed for neutral atomic targets. 

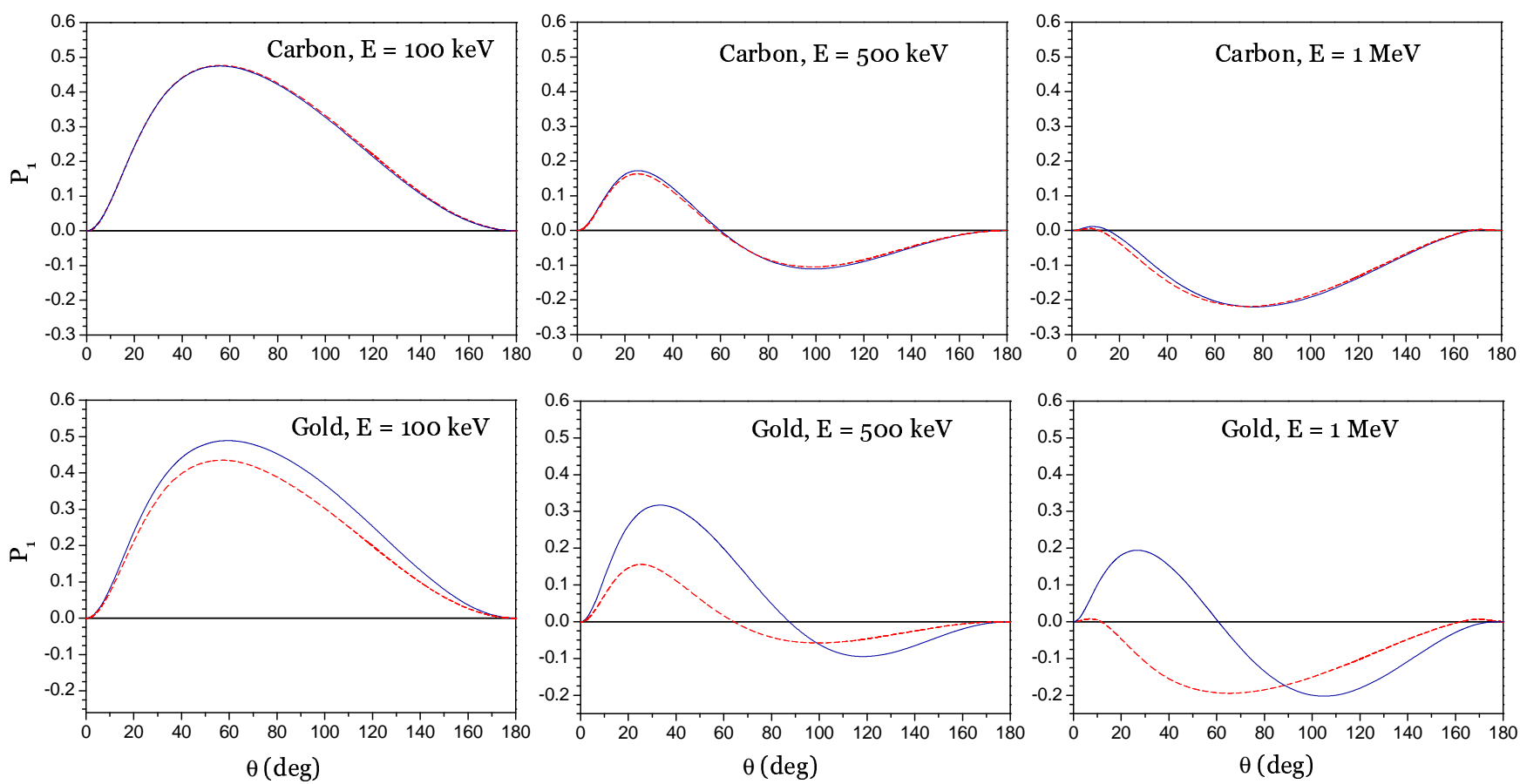

FIG. 3: (Color online) Stokes parameter $P_{1}$ for the initially unpolarized electrons (blue, solid line) and positrons (red, dashed line), for the carbon (top) and gold (bottom) targets, as a function of the photon emission angle, for different initial energies of the projectile $E$. The fractional energy carried by photon is $k / E=0.5$.
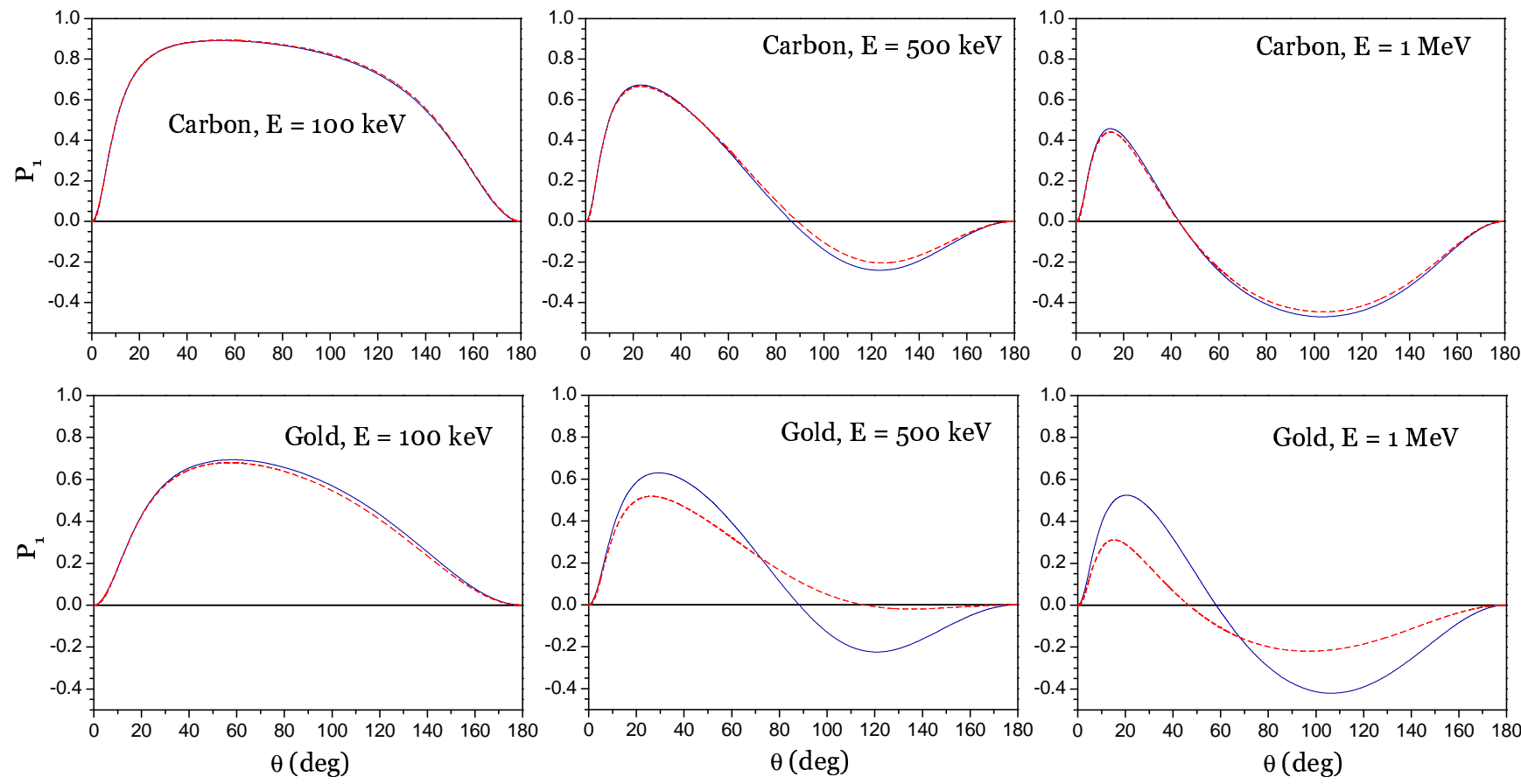

FIG. 4: (Color online) Stokes parameter $P_{1}$ for the initially unpolarized electrons (blue, solid line) and positrons (red, dashed line), for the carbon (top) and gold (bottom) targets, as a function of the photon emission angle, for different initial energies of the projectile $E$. The fractional energy carried by photon is $k / E=0.9$. 

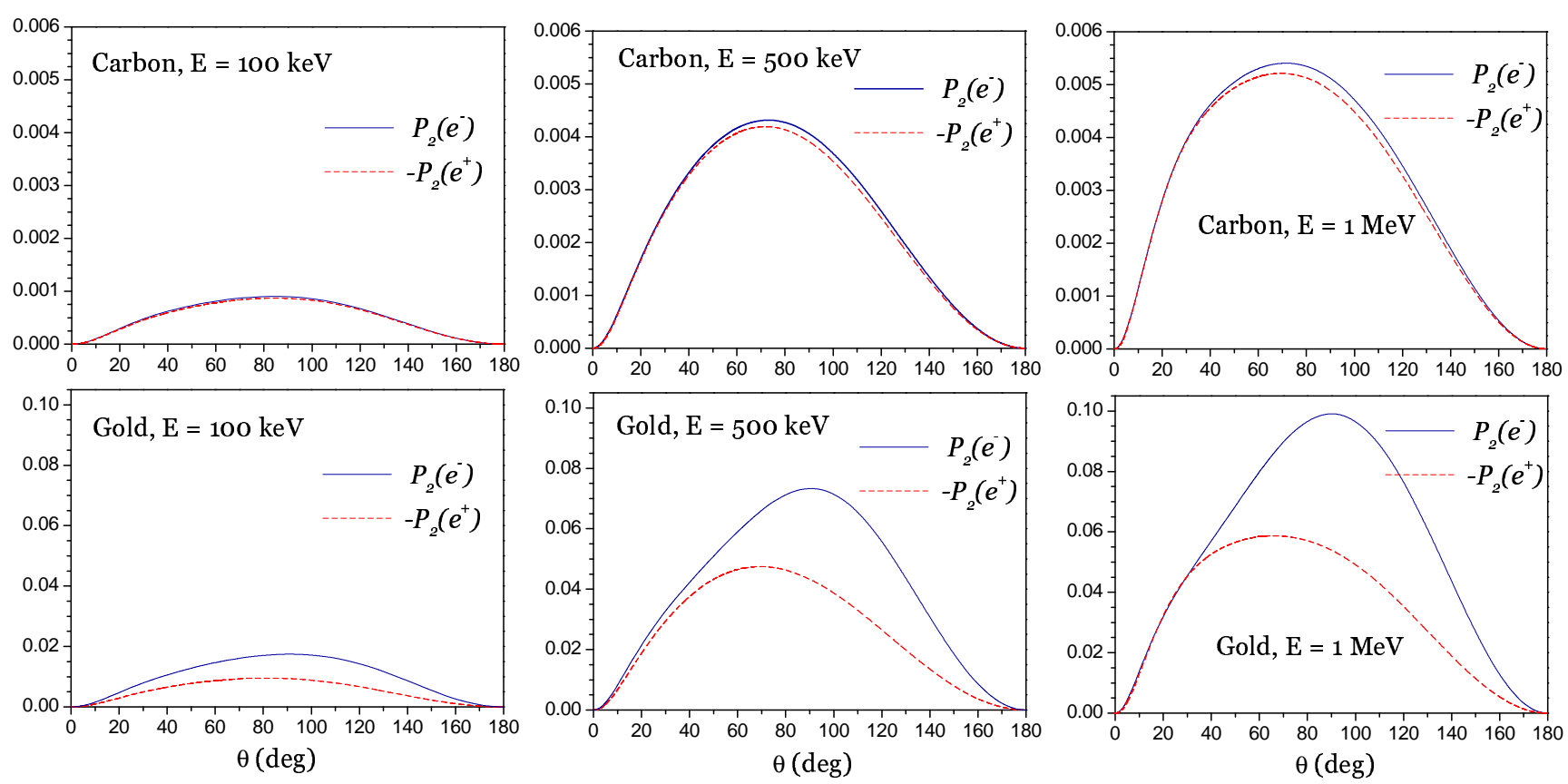

FIG. 5: (Color online) Stokes parameter $P_{2}$ for the initially logitudinally polarized electrons $\left(P_{2}\left(e^{-}\right)\right.$, blue, solid line) and positrons $\left(P_{2}\left(e^{+}\right)\right.$, red, dashed line), for the carbon (top) and gold (bottom) targets, as a function of the photon emission angle, for different initial energies of the projectile $E$. The fractional energy carried by photon is $k / E=0.5$. Note that, for positrons, $P_{2}$ with the reversed overall sign is plotted. 